\title{
Coupling Current and AC Loss in LHC Superconducting Quadrupoles
}

\author{
Mario Di Castro, Luca Bottura, David Richter, Stephane Sanfilippo, and Rob Wolf
}

\begin{abstract}
One of the issues for the operation of the LHC accelerator at CERN are the field errors generated by coupling currents in the superconducting cables of the main dipoles and quadrupoles, especially during the initial phase of the energy ramp from injection conditions. Coupling current effects have already been measured in the superconducting dipoles, and results are reported elsewhere. This paper reports similar measurements that we have recently performed on different types of LHC superconducting quadrupoles (arc quadrupole, dispersion suppressor and matching section quadrupoles) to quantify the above effects and compare them to the values specified from the beam tolerances. Loss and field errors due to ramping are mainly determined by the contact resistance $R_{c}$ between the strands of the magnets cables. In this paper the $R_{c}$ is calculated for several quadrupoles measured using both the measured energy loss and the magnetic field errors during ramping of magnets.
\end{abstract}

Index Terms-Author, please supply your own keywords or send a blank e-mail to keywords@iee.org to receive a list of suggested keywords.

\section{INTRODUCTION}

$\mathbf{T}$ THE Large Hadron Collider (LHC) now under installation at CERN will provide proton-proton collisions with a center-of-mass energy of $14 \mathrm{TeV}$. The coils of the superconducting accelerator magnets of the LHC are made with Rutherford type cables consisting of two layers of twisted strands. During the particle acceleration ramp, the magnetic field variation in the coil induces coupling currents (eddy currents) circulating among the strands of the superconducting cables as well as among the filaments of the strands. In the regime of interest for accelerator operation (slow ramps) the intensity of the induced currents is proportional to the ramp-rate. Coupling currents among strands in the cable are inversely proportional to the contact resistance Rc between crossing strands. In the strands, coupling currents among filaments are proportional to the strand time constant $\tau$. One of the issues associated with coupling currents is that they generate a field contribution in the magnet bore [1] that, in principle, has to be evaluated, measured and must be taken into account in the synchronization of the power converters that drive the main dipoles and quadrupoles [2]. The field error associated to the coupling currents appears as a field advance (FA), meaning that the contribution of the eddy current to the main field component is positive: the field is larger than expected in the magnet bore. The second issue associated with coupling currents are AC

Manuscript received August 25, 2007.

The authors are with CERN, Geneva, Switzerland (e-mail: mariodicastro@gmail.com).

Color versions of one or more of the figures in this paper are available online at http://ieeexplore.ieee.org.

Digital Object Identifier 10.1109/TASC.2008.921305 losses. Losses can lead to a quench because of over-heating if the value of $R_{c}$ is too low. On the other hand, the cross contact resistance has an influence on the current redistribution among strands over lengths longer than the twist pitch, that also takes place during the field change. In this respect, high values of $\mathrm{R}_{\mathrm{c}}$ can lead to non-uniform current distribution and pre-mature quench. The coupling current effect has been measured in the past for the bending dipoles and has been found to be small [3]. The measured dipole field advance is about 2 units (1 unit is equal to $10^{-4}$ of the main field), while the ramp rate effect on multipoles is negligible. These results are consistent with inter-strand resistance values $R_{c}$ in excess of $50 \mu \Omega$ estimated from AC loss measurements. To limit the field errors in the LHC main quadrupole, we require an $\mathrm{R}_{\mathrm{c}}$ greater than $20 \mu \Omega$, and conductance differences smaller than 0.1 MS between parts of the magnet coils [4]. This value represents a good compromise between field advance and multipole errors (small), AC loss (small) and premature quenches due to current distribution (not an issue at the required ramp-rate). This paper describes the measurements of the FA, of the ramp rate induced field error and also of the AC loss performed on several main quadrupoles of the Short Straight Sections (SSS) tested at CERN in cold conditions (1.9 K) during 2006 and the first months of 2007. An overview of the measurement system and of the data treatment technique is presented for each type of measurements. We report the field advance of the main field $\left(\mathrm{B}_{2}\right)$, the ramp rate effect on multipoles $\left(c_{n}\right)$ and the AC loss measured. The cables inter-strand resistance $R_{c}$ is deduced using both the measured loss and field errors during current ramping of magnets.

\section{Measurement Techniques and Data Processing}

\section{A. Ramp Rate Effects on Main Field}

The measurements, performed at the same time for both apertures, were conducted on eight main quadrupoles using five stationary coils placed in the center of each aperture and connected in series [5]. The variation of field $\Delta \mathrm{B}_{2}$ between the ramp up and the ramp down was obtained from the integral of the voltage induced in the coils during current cycles, ranging from $1000 \mathrm{~A}$ to $5000 \mathrm{~A}$ and performed with different ramp rates from 10 to $50 \mathrm{~A} / \mathrm{s}$. The core of the acquisition system is a series of VME-PDI's (Precision Digital Integrators) triggered at $1 \mathrm{~Hz}$ by an external, frequency controlled function generator used to eliminate the difference between the integration time and the current given by the power supply [3]. The acquisition was connected to the coil "A" (top) of 5 coil sectors centered with respect to the magnet length. The voltage measured by each integrator is divided by the amplification gain; then the voltage offset is computed and removed from the coils signal. The voltage offset is in the range of $1.5-2 \mu \mathrm{V}$ and is mostly due to the input amplifier. However, it is constant and stable within 


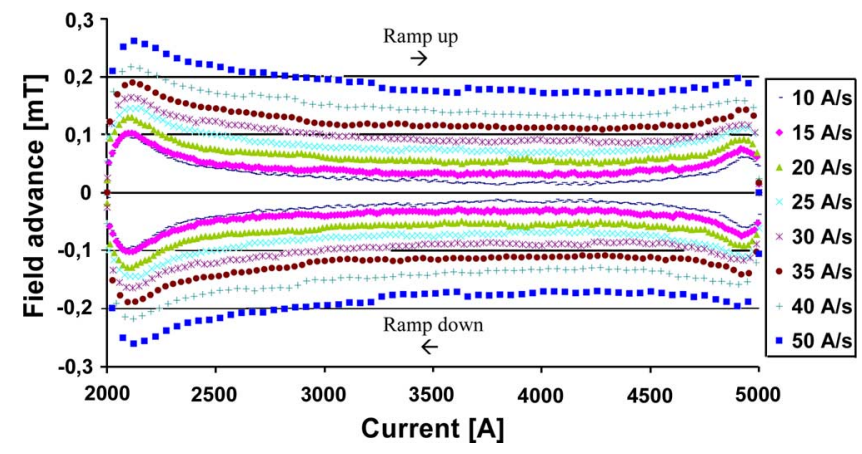

Fig. 1. Magnetic field advance in a quadrupole as a function of current at different ramp rate.

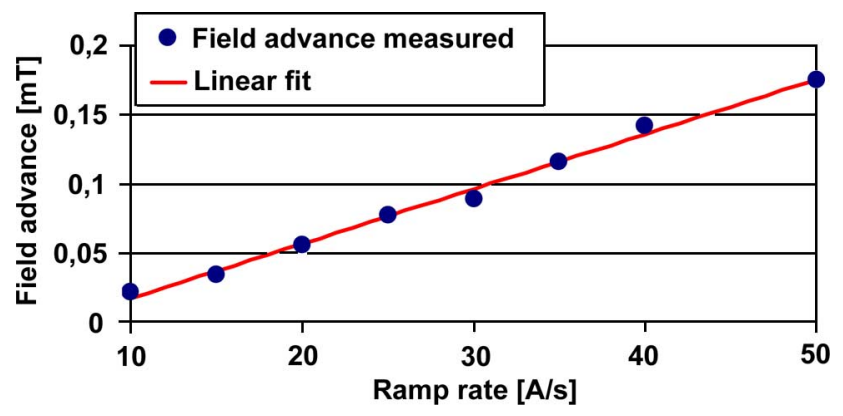

Fig. 2. Field advance dependence on the ramp rate averaged in the linear zone [3000-4000 A] on a coil sector.

the noise level during the entire measurement. This feature allows a precise and reliable software correction of the offset. The magnetic flux $\varphi$ for each coil is calculated as the time integral of the induced voltage corrected for the integrator offset:

$$
\varphi_{i}=-\int V_{i} d t
$$

The main magnetic field for each coil $\left(\mathrm{B}_{\mathrm{i}}\right)$ is calculated by dividing the magnetic flux by the surface area $\left(A_{i}\right)$ of the coil:

$$
B_{i}=\frac{\varphi_{i}}{A_{i}}
$$

An example of measured field is reported in Fig. 1, where we have removed the linear field contribution for clarity of presentation. In order to distinguish the ramp-rate effects from the steady-state contribution, we compare the field measured at different ramp-rates dI/dt. The half amplitude of the field hysteresis $\Delta \mathrm{B}$ is computed from the difference of the measured field at a given current $\mathrm{I}_{\mathrm{k}}$ during ramp-down $\mathrm{B}^{\text {down }}\left(\mathrm{I}_{\mathrm{k}}\right)$ and ramp-up $\mathrm{B}^{\mathrm{up}}\left(\mathrm{I}_{\mathrm{k}}\right)$ (at a given ramp-rate):

$$
\Delta B_{n}\left(I_{k}\right)=\frac{1}{2}\left(B_{n}^{u p}\left(I_{k}\right)-B_{n}^{\text {down }}\left(I_{k}\right)\right) .
$$

The field hysteresis $\Delta \mathrm{B}$ is then plotted as a function of the ramp-rate dI/dt (see Fig. 2). The slope $\mathrm{S}$ of the $\Delta \mathrm{B}(\mathrm{dI} / \mathrm{dt}$ ) is the ramp rate field contribution at the current $I_{k}$. The calculation is repeated at several values of $\mathrm{I}_{\mathrm{k}}$ in the current range 3-4 kA, where the hysteresis is relatively constant(see Fig. 1) and then averaged. The dependence of $\Delta \mathrm{B}_{2}$ on the ramp rate can be written as:

$$
\Delta B_{2}=\Delta B_{2}^{(0)}+S \frac{d I}{d t} .
$$

The first term is a negative offset corresponding to the effect of the demagnetization. The coefficient $\mathrm{S}$ reflects mostly the interstrand coupling although a small correction for inter-filament coupling is necessary and will be discussed later.

\section{B. Ramp Rate Effects on Multipoles}

Current ramps induce field distortions for all the harmonics [6]. Like the field advance analysis, in order to distinguish the ramp-rate effects from the steady-state contribution, we compare the multipoles field measured at a different ramp-rate $\mathrm{dI} / \mathrm{dt}$. To determine the $\mathrm{AC}$ contributions to the field errors, measurements with the 6-m long rotating coils [7] were carried out in both apertures during ramps between injection $\mathrm{I}_{\text {inj }}(760 \mathrm{~A})$ and nominal currents $\mathrm{I}_{\text {nom }}(11850 \mathrm{~A})$ at different ramp rates ranging from 10 to $50 \mathrm{~A} / \mathrm{s}$ using a step of $10 \mathrm{~A} / \mathrm{s}$. The analysis is done separately for each normal and skew harmonic. The multipoles hysteresis $\Delta B_{n}(n>2)$ is calculated using (3) for each ramp-rate $\mathrm{dI} / \mathrm{dt}$. The slope $\mathrm{S}$ of the $\Delta \mathrm{B}_{\mathrm{n}}(\mathrm{d} \mathrm{I} / \mathrm{dt})$ is the ramp rate field contribution at the current $\mathrm{I}_{\mathrm{k}}$. The calculation is repeated at several values of $I_{k}$ in the current range 4000-9000 A where the hysteresis is relatively constant, and then averaged.

\section{AC Energy Loss}

During charging and discharging of the superconducting magnets energy is dissipated through different mechanisms. The main contributions are due to the magnetization of the superconducting filaments and to the coupling currents between the strands in the cables and between the filaments in the strands. Smaller contributions are caused by the ohmic loss in the connection between the poles and in the splices between the inner and outer layer, the magnetization of the iron yoke and the eddy currents in the copper spacers and in the collars [8]. Loss measurements can produce the same information as the field advance measurements as $R_{c}$ can be also recomputed from the coefficient of loss dependence of the ramp rate. Loss experiments on the quadrupoles were therefore conducted at $1.9 \mathrm{~K}$ ramping the magnets by using standard current cycles. The current is changing from $I_{\min }=1000 \mathrm{~A}$ to $\mathrm{I}_{\max }=4000 \mathrm{~A}$ and back to $I_{\min }$ at the ramp rates $\mathrm{dI} / \mathrm{dt}$ ranging from 20 to 90 A/s [9]. Six multi-meters Keithley 2001 were used and each one recorded one channel: the current I, the voltage $\mathrm{V}$ across the whole magnet, the voltage across each aperture, the voltage across the pole $1+2$ and the voltage across the pole 3-4 of one of the two apertures. The sampling time for the current acquisition and for the integration time of the multimeters was set equal to $200 \mathrm{~ms}$. After each cycle, the total energy loss $\mathrm{W}_{\text {total }}$ was calculated in the entire magnet, while the values $\mathrm{W}_{1}$ and $\mathrm{W}_{2}$ are related to the loss in the first and the second aperture respectively. The offset and the joint resistance effects were removed from the recorded voltage signals. The total energy loss across the whole magnet and the energy loss for each aperture were obtained by integrating the product between 
TABLE I

Measured Values of the Slope S (Average Value of the Central COILS) AND THE FA FOR EIGHT MAIN QUADRUPOLE

\begin{tabular}{lcccc}
\hline \hline SSS & Aperture & $\begin{array}{c}\text { S } \\
{[\mu \mathrm{Ts} / \mathrm{A}]}\end{array}$ & $\begin{array}{c}\text { Sigma S } \\
{[\mu \mathrm{Ts} / \mathrm{A}]}\end{array}$ & $\begin{array}{c}\text { B2 Field Advance } \\
{\left[\text { units@ } @ \mathrm{R}_{\mathrm{ref}} @ 760 \mathrm{~A} @ 10 \mathrm{~A} / \mathrm{s}\right]}\end{array}$ \\
\hline 317 & 1 & 2.66 & 0.593 & 1.0 \\
317 & 2 & 3.75 & 0.591 & 1.5 \\
318 & 1 & 4.53 & 0.691 & 1.7 \\
318 & 2 & 4.95 & 0.296 & 1.8 \\
361 & 1 & 6.17 & 0.335 & 2.4 \\
361 & 2 & 4.25 & 0.458 & 1.6 \\
366 & 1 & 3.26 & 0.256 & 1.2 \\
366 & 2 & 2.93 & 0.302 & 1.1 \\
374 & 1 & 5.13 & 0.305 & 2.0 \\
374 & 2 & 4.55 & 0.347 & 1.7 \\
520 & 1 & 5.11 & 0.301 & 2.0 \\
520 & 2 & 4.57 & 0.317 & 1.7 \\
525 & 1 & 3.75 & 0.041 & 1.5 \\
525 & 2 & 4.12 & 0.320 & 1.6 \\
529 & 1 & 4.11 & 0.065 & 1.6 \\
529 & 2 & 4.20 & 0.046 &
\end{tabular}

the current and the voltage for the total time $t_{c}$ of each current cycle at different ramp rates:

$$
W=\int_{0}^{t_{c}} V I d t
$$

The dependence $\mathrm{W}=\mathrm{W}(\mathrm{dI} / \mathrm{dt})$ can be fitted using the linear expression:

$$
W=W_{h}+2 k\left(I_{\max }-I_{\min }\right) \frac{d I}{d t},
$$

where $\mathrm{W}_{\mathrm{h}}=\mathrm{W}(0)$ is the hysteresis energy loss.

\section{EXPERIMENTAL RESULTS VS EXPECTED ONES}

\section{A. Ramp Rate Effects on the Main Field}

The main result of the analysis is the quantity $\mathrm{S}$, representing the slope of the plot of the half field hysteresis $\Delta \mathrm{B}$ vs. the current ramp-rate dI/dt. Fig. 1 shows an example of the field measured for the SSS525, where we have removed the linear field component, proportional to the current, for clarity of presentation. In Fig. 2 we show the dependence of the hysteresis $\Delta \mathrm{B}$ on the ramp rate $\mathrm{dI} / \mathrm{dt}$, that has an approximate linear dependence as expected from (4).

The measured slopes $\mathrm{S}$ in Ts/A for eight main quadrupoles are presented in Table I as well as the field advance at $10 \mathrm{~A} / \mathrm{s}$ and injection field (760 A), averaged over the three central coils of each aperture. The standard deviation of the slope values of the three central coils for each aperture is steady at about one order of magnitude less than the average value. From Table I the estimated field advance is in the range of 1.0-2.4 units. This effect is much smaller than the predicted 15 units calculated for an inter-strand resistance of $20 \mu \Omega$ [10].

The mean value of the Field Advance is used to estimate the inter-strand resistance $R_{c}$ :

$$
R_{c}=\frac{k_{B}^{O L} L_{P}^{O L}+k_{B}^{I L} L_{P}^{I L}}{d B / d(d I / d t)}
$$

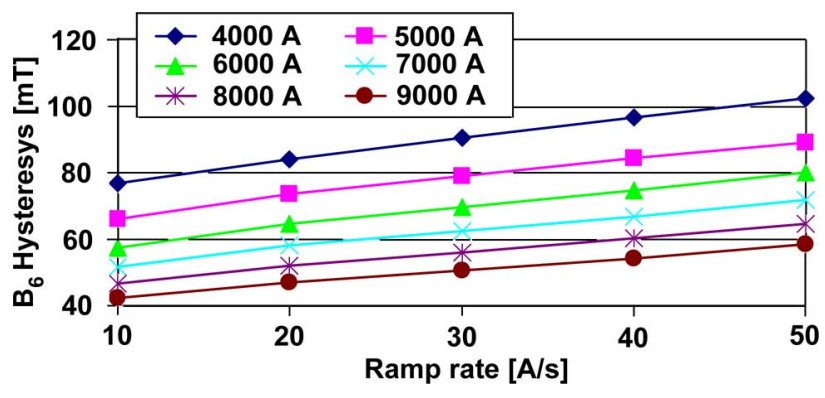

Fig. 3. The $\mathrm{B}_{6}$ Hysteresis for the second aperture of the SSS317. The ramp rate effect is calculated as the average value of the slope measured for each current (from $4 \mathrm{kA}$ to $9 \mathrm{kA}$ ).

TABLE II

AVERAGE AND STANDARD DEVIATION OF THE RAMP RATE INDUCED

\begin{tabular}{|c|c|c|}
\hline $\begin{array}{c}\text { Multipoles } \\
{[-]} \\
\end{array}$ & $\begin{array}{l}\text { AC average magnetization } \\
\text { [units@R } \mathrm{R}_{\text {ref } @ 760 \mathrm{~A} @ 10 \mathrm{~A} / \mathrm{s}]}\end{array}$ & $\begin{array}{c}\text { Sigma AC magnetization } \\
{\left[\text { units@R } \mathrm{R}_{\mathrm{ref}} @ 760 \mathrm{~A} @ 10 \mathrm{~A} / \mathrm{s}\right]}\end{array}$ \\
\hline b3 & 0.206 & 0.072 \\
\hline b4 & 0.080 & 0.007 \\
\hline b6 & 0.022 & 0.018 \\
\hline a3 & 0.170 & 0.085 \\
\hline a4 & 0.150 & 0.035 \\
\hline
\end{tabular}
Multipoles MEAsured AT COLD CONDITIONS ON 14 MAIN QuAdRUPOLE APERTURES

where $\mathrm{Lp}$ are the twist pitches of the outer and inner layers and $\mathrm{k}_{\mathrm{B}}$ outer and inner layers field factors coming from a numerical simulation with a theoretical field advance produced by an inter-strand resistance of $1 \mu \Omega$. The resulting inter-strand resistance calculated using the FA measurements is in the range of 95-230 $\mu \Omega$ for the three central coils.

\section{B. Ramp Rate Effects on Multipoles}

Fig. 3 shows an example of hysteresis for the $\mathrm{B}_{6}$ at different ramp rates for 6 different $I_{k}$ in the current range chosen.

In Table II the average results of the ramp analysis measurements performed on seven main quadrupoles are presented for the harmonics $b_{3}, b_{4}, b_{6}, a_{3}, a_{4}$. The results are given in units at reference radius $\left(R_{\text {ref }}\right)$ of $17 \mathrm{~mm}$, at injection current (760 A) and at a reference current ramp rate of $10 \mathrm{~A} / \mathrm{s}$ The results can be compared to the expected field errors calculated for an inter-strand resistance of $20 \mu \Omega$.

The field distortion coming from the ramp rate is weak. The measured ramp rate normal multipoles are less than 0.2 units at injection and correspond to an inter-strand resistance of about $135 \mu \Omega( \pm 70 \mu \Omega)$. The multipoles values are smaller than the expected ones, in agreement with values of $R_{c}$ well above $20 \mu \Omega$.

\section{AC Energy Loss}

The AC energy loss measurements were performed on 3 quadrupoles (six apertures); a hysteresis loss of $\mathrm{W}_{\mathrm{h}}=(140-$ $180) \mathrm{J}$ and a coefficient $\mathrm{k}=(8.7 \mathrm{E}-05-9.9 \mathrm{E}-05) \mathrm{Js} / \mathrm{A}^{2}$ ((6)) were found. The $\mathrm{k}$ coefficient allows [8] the average contact resistance of the cable $R_{c}=(150-200) \mu \Omega$ to be calculated. These estimations do not take into account the inter-filament contribution but only the inter-strand contribution. A correction can be done assuming the average time 
TABLE III

Results of THE AC ENERgy Loss MEASUREMENTS PERFoRMED ON THREE MAIN QUADRUPOLES

\begin{tabular}{|c|c|c|c|c|c|c|}
\hline SSS & $\begin{array}{c}\text { k Ap.1 } \\
{\left[\mu \mathrm{J} \mathrm{s} / \mathrm{A}^{2}\right]}\end{array}$ & $\begin{array}{c}\text { k Ap.2 } \\
{\left[\mu \mathrm{J}_{\mathrm{s}} / \mathrm{A}^{2}\right]}\end{array}$ & $\begin{array}{c}\text { Re Ap.1 } \\
{[\mu \Omega]}\end{array}$ & $\begin{array}{c}\text { Rc Ap.1 } \\
\text { corrected } \\
{[\mu \Omega]}\end{array}$ & $\begin{array}{c}\text { Re Ap.2 } \\
{[\mu \Omega]}\end{array}$ & $\begin{array}{c}\text { Rc Ap. } 2 \\
\text { corrected } \\
{[\mu \Omega]}\end{array}$ \\
\hline 361 & 92.9 & 87.1 & 163 & 169 & 174 & 181 \\
\hline 374 & 98.9 & 79.5 & 154 & 159 & 189 & 198 \\
\hline 529 & 91.1 & 91.8 & 167 & 173 & 165 & 171 \\
\hline
\end{tabular}

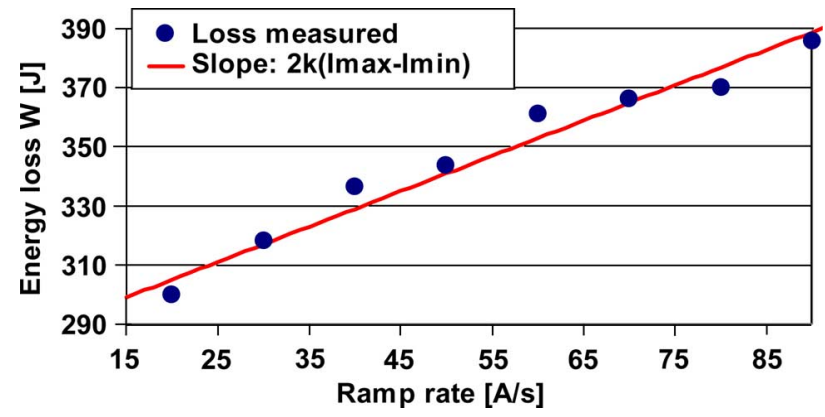

Fig. 4. The $\mathrm{W}=\mathrm{W}(\mathrm{dI} / \mathrm{dt})$ dependence for the SSS529, aperture \#1.

TABLE IV

COMPARISON BETWEEN THE $R_{c}$ MEASURED USING FA AND AC ENERgy LosS MEASUREMENTS AND THE EXPECTED VALUES

\begin{tabular}{ccccc}
\hline \hline SSS & Aperture & $\begin{array}{c}\text { Rc from FA } \\
\text { Measurement } \\
{[\mu \Omega]}\end{array}$ & $\begin{array}{c}\text { Rc from Loss } \\
\text { Measurement } \\
{[\mu \Omega]}\end{array}$ & $\begin{array}{c}\text { Range of } \\
\text { Expected Values } \\
{[\mu \Omega]}\end{array}$ \\
\hline 317 & 1 & 230 & $\# \#$ & $130-310$ \\
317 & 2 & 153 & $\# \#$ & $30-120$ \\
318 & 1 & 135 & $\# \#$ & $30-120$ \\
318 & 2 & 128 & $\# \#$ & $70-210$ \\
361 & 1 & 96 & 169 & $50-150$ \\
361 & 2 & 144 & 181 & $60-220$ \\
366 & 1 & 192 & $\# \#$ & $\# \#$ \\
366 & 2 & 208 & $\# \#$ & $20-120$ \\
374 & 1 & 115 & 159 & $\# \#$ \\
374 & 2 & 135 & 198 & $150-320$ \\
520 & 1 & 115 & $\# \#$ & $\# \#$ \\
520 & 2 & 135 & $\# \#$ & $70-220$ \\
525 & 1 & 153 & $\# \#$ & $70-210$ \\
525 & 2 & 144 & $\# \#$ & $\# \#$ \\
529 & 1 & 144 & 173 & $\# \#$ \\
529 & 2 & 144 & 171 & $50-130$ \\
\hline \hline
\end{tabular}

constant $n \tau=0.03 \mathrm{~s}$ for the strands. However, this figure is a rough estimate because the time constant could deviate significantly from the average value. Table III summarizes the results of $\mathrm{AC}$ loss measurements and the calculation of the $R_{c}$ with and without the inter-filament correction. Loss measurements confirm that the $R_{c}$ value is well above the specified value of 20 $\mu \Omega$ and the results are consistent with the $R_{c}$ values calculated using the ramp rate measurements. An example of energy loss at different ramp rates for the first aperture of the SSS529 is illustrated in Fig. 4.

Several measurements were conducted in the past in static conditions on cables samples trying to estimate a range of expected values of the cables cross contact resistance [11]. In Table IV is shown a comparison between the measured values of $R_{c}$ obtained with different measurement techniques (Field Advance and Energy Loss) and the expected values. The measurement uncertainty for the FA is 0.1 units $(25 \mu \Omega$ for the $\mathrm{R}_{\mathrm{c}}$ ) while for the AC energy loss is $1.5 \mu \mathrm{J}$ ( $5 \mu \Omega$ for the $\left.R_{c}\right)$. The resulting $R_{c}$ measured is more or less in the range of the expected values measured on cable samples. The minimum values of the range of the expected $R_{c}$ are measured on cables samples at $190 \mathrm{C}$ after $30 \mathrm{~min}$ of curing while the maximum values correspond to measurements before the curing.

\section{CONCLUSIONS}

We have found a reliable measurement method to calculate the coupling current effect on the main LHC superconducting quadrupoles. The field errors induced by the ramp rate on the multipoles are generally small and about the same order of magnitude as the resolution of the measurement. For the measurement of the Field Advance, with the proper choice of trigger time (of the order of $1 \mathrm{~s}$ ) the acquisition system is capable to measure a ramp-rate dependent field within a $\mu \mathrm{T} / \mathrm{A} / \mathrm{s}$ accuracy. This corresponds to an error of the order of 0.1 units of main field at injection condition and $10 \mathrm{~A} / \mathrm{s}$ ramp-rate, and hence fully sufficient for the characterization of the dipole and quadrupole field quality. The Field Advance measured on eight quadrupoles is found to be small, on the order of 1-2.4 units at injection current $(760 \mathrm{~A})$. These results lead to an estimate of the inter-strand resistance being of order of 95-230 $\mu \Omega$. This order of magnitude of $R_{c}$ is consistent with the results coming from the AC energy loss measurements and with the expected values coming from measurements performed on cable samples. The quadrupoles $R_{c}$ values measured are suitable for the operation of the LHC.

\section{REFERENCES}

[1] R. Wolf et al., "Determination of interstrand contact resistance from loss and field measurements in LHC dipole prototypes and correlation with measurements on cable samples," IEEE Trans. Appl. Supercond., vol. 7, no. 2, Jun. 1997.

[2] T. Ogitsu, A. Devred, and V. Kovachev, "Influence of inter-strand coupling current on field quality of superconducting accelerator magnets," Part. Accel., vol. 57, pp. 215-235, 1997.

[3] L. Bottura et al., Engineering Report, Measurement of Cable and Strand Coupling Currents in the MBB-A002 Using the AT-680-2030-050 PDI EDMS 322099, 2001.

[4] J. D. Adam et al., "Status of the LHC superconducting cable mass production," presented at the 17th International Conference on Magnet Technology, Geneva, Switzerland, Sep. 2001.

[5] M. Di Castro et al., Ramp Rate Effects, AC Energy Loss and Indirect Rc Measurements on Main Quadrupoles in the Short Straight Sections CERN internal note, AT-MTM-IN-2007-003, EDMS 831181.

[6] L. Bottura et al., "Field quality of the LHC dipole magnets in operating conditions," in Proceedings of EPAC 2002, Paris, France.

[7] J. Billan and L. Bottura et al., "Twin rotating coils for cold magnetic measurements of $15 \mathrm{~m}$ long LHC dipoles," IEEE Trans. Applied Superconductivity, March 2000.

[8] A. Verweij et al., "Analysis of the AC Loss measurements on the onemetre dipole model magnets for the CERN LHC," IEEE Trans. Magn., vol. 30, no. 4, Jul. 1994.

[9] I. Bejar et al., "Measurement of AC loss and magnetic field during ramps in the LHC model dipoles," IEEE Trans. Appl. Supercond., vol. 9, no. 2, June 1999.

[10] A. Akhmetov, "AC loss in a stack of flat supercondacting cables," IEEE Trans. Appl. Supercond., vol. 12, March 2002.

[11] D. Richter, J. D. Adam, J.-M. Depond, D. Leroy, and L. R. Oberli, "DC Measurement of electrical contacts between strands in superconducting cables for the LHC main magnets," IEEE Trans. Appl. Supercond., vol. 7, no. 2, pp. 786-792, 1997. 